\title{
Overexpression of the secretory small GTPase Rab27B in human breast cancer correlates closely with lymph node metastasis and predicts poor prognosis
}

Jia-Xing Zhang ${ }^{1 \dagger}$, Xiao-Xia Huang ${ }^{1,2 \dagger}$, Man-Bo Cai ${ }^{3 \dagger}$, Zhu-Ting Tong ${ }^{4}$, Jie-Wei Chen², Dong Qian ${ }^{1}$, Yi-Ji Liao', Hai-Xia Deng ${ }^{1}$, Ding-Zhun Liao ${ }^{2}$, Ma-Yan Huang ${ }^{2}$, Yi-Xin Zeng ${ }^{1}$, Dan Xie ${ }^{1,2^{*}}$ and Shi-Juan Mai ${ }^{1^{*}}$

\begin{abstract}
Background: The secretory small GTPase Rab27b was recently identified as an oncogene in breast cancer (BC) in vivo and in vitro studies. This research was designed to further explore the clinical and prognostic significance of Rab27B in BC patients.

Methods: The mRNA/protein expression level of Rab27B was examined by performing Real-time PCR, western blot, and immunohistochemistry $(\mathrm{IHC})$ assays in 12 paired BC tissues and matched adjacent noncancerous tissues (NAT). Then we carried out IHC assay in a large cohort of 221 invasive BC tissues, 22 normal breast tissues, 40 fibroadenoma (FA), 30 ductual carcinoma in situ (DCIS) and 40 metastatic lymph nodes (LNs). The receiver operating characteristic curve method was applied to obtain the optimal cutoff value for high Rab27B expression. Epithelial-mesenchymal transition (EMT) marker expression levels were detected in relation to Rab27B expression.

Results: We observed that the increased expression of Rab27B was dependent upon the magnitude of cancer progression $(P<0.001)$. The elevated expression of Rab27B was closely correlated with lymph node metastasis, advanced clinical stage, ascending pathology classification, and positive ER status. Furthermore, patients with high expression of Rab27B had inferior survival outcomes. Multivariate Cox regression analysis proved that Rab27B was a significantly independent risk factor for patients' survival $(P<0.001)$. Furthermore, a significant positive relationship was observed between Rab27B expression and elevated mesenchymal EMT markers.
\end{abstract}

Conclusion: Our findings suggest that overexpression of Rab27B in BC coincides with lymph node metastasis and acquisition of a poor prognostic phenotype.

Keyword: Rab27B, Breast cancer, Prognosis, EMT, Metastasis

\section{Background}

Breast cancer (BC) is by far the leading malignancy in women and is a serious threat to the health of women worldwide [1-3]. Despite the great advance achieved in therapy technology recently, the prognosis of the cancer remains unsatisfactory in the clinic [4]. Substantial effort

\footnotetext{
* Correspondence: xiedan@sysucc.org.cn; maishj@sysucc.org.cn

${ }^{\dagger}$ Equal contributors

'State Key Laboratory of Oncology in South China, Cancer Center, Sun Yat-

Sen University, 651\# Dongfeng Road East, Guangzhou 510060, China

2Department of Pathology, Cancer Center, Sun Yat-Sen University, 651\#

Dongfeng Road East, Guangzhou 510060, China

Full list of author information is available at the end of the article
}

has focused on the gene alterations and specific molecular markers that are responsible for the tumorigenesis and progression of this malignancy. Because of prognostic markers, including estrogen receptor (ER), progesterone receptor (PR), CerbB-2, carcinoembryonic antigen, CA 15-3, CA 27.29, the risk classification of a BC patient's outcome can be defined more accurately. Thus, identification of more biomarkers that could be used to define the progression of this malignancy may be of great benefit to BC patients [5]. However, the identification of promising molecular biomarkers in $\mathrm{BC}$ that have clinical significance is still substantially limited.

\section{Biomed Central}


Rab GTPases, which function as molecular switches that alternate between active GTP-bound and inactive GDP-bound conformational states, constitute the largest family of small GTPases and play a vital role in endocytosis and exocytosis vesicle-trafficking control [6-9]. When Rabs are activated, the vesicles are engaged to specific effectors required for vesicle movement, docking, and fusion [10]. The secretory Rab GTPases, including Rab26, Rab37, Rab3A/B/C/D, and Rab27A/B, are reported to be responsible for regulated vesicle exocytosis [6]. The Rab27 subfamily, including the homologues Rab27A and B, are 71\% identical at the amino acid level and are present in several secretory tissues and hematopoietic cells [11-14].

Rab proteins of both the endocytic and exocytosis pathways play critical roles in cancer progression [15-22]. Recently, Rab27b was reported to regulate invasive growth and metastasis in ER-positive BC cell lines in vitro and in vivo [23]. Also, the presence of Rab27B protein was observed to be associated with a low degree of differentiation and the presence of lymph node metastasis in ER-positive BC. However, this study was limited by its small sample size. Moreover, without adequate patient survival data, we could not investigate the effect of Rab27B on patient prognosis. Thus, the final conclusion in this research did not seem convincing.

Our study was designed to fully investigate Rab27B's expression pattern in $\mathrm{BC}$ as well as its relationship with clinical parameters and survival prognosis. We enrolled $221 \mathrm{BC}$ patients with defined clinicopathologic features to investigate the prognostic value of Rab27B in BC. Our findings strongly suggest that elevated expression of Rab27B may be a risk factor that is predictive of prognosis in $\mathrm{BC}$ patients following appropriate therapy.

\section{Materials and methods}

\section{Patients, tissue specimens and follow up produce}

Formalin-fixed, paraffin-embedded tissues (FFPET) of 221 invasive BC samples obtained from 221 women were histologically and clinically diagnosed at the Cancer center, Sun Yat-Sen University (SYSUCC) from January 2000 to December 2002. Additionally, 22 specimens of normal breast tissues from exairesis for non-breast diseases, 40 fibroadenoma of breast (FA), 30 Ductal carcinoma in situ (DCIS) and 40 corresponding metastatic lymph nodes(LNs) were also obtained from the patients in SYSUCC as control. All patients were classified according to the American Joint Committee on Cancer (AJCC) and tumor node metastasis (TNM) classification systems [24]. FFPETs of 221 surgical patients were used to construct the tissue microarray that was used for immunohistochemical (IHC) staining [25,26]. Fourteen 12 Pairs of $\mathrm{BC}$ tissues and matched adjacent noncancerous tissues (NAT) were frozen and stored in liquid nitrogen until further use. All samples that we selected were not subjected to preoperative radiotherapy and/or chemotherapy. This study was approved by the Research Ethics Committee of SYSUCC. The patients were followed every 3 months for the first year and then every 6 months for the next 2 years and finally annually. The total disease-specific survival (DSS) follow-up period was defined as the time from operation to the date of cancer-related death or when censured at the lasted date if patients were still alive. All patients still alive at the time of analysis have reached a minimum follow-up period of 60 months (median: 79.0 months; range 60.0 to 112.0 months). A total of 51 patients died from cancer related death during follow-up period.

\section{Immunohistochemistry staining (IHC)}

We used the Dako Real Envision Kit (K5007, Dako) for IHC staining analysis. Hormonal receptors were evaluated with the 1D5 antibody for estrogen receptor a (ERa) and antibody PGR-1A6 for progesterone receptor (PR; Dako). CerbB2 was detected with CB11 (Dako). Only tumor tissues with distinct nuclear staining for ER and PR in $>10 \%$ of the tumor cells were recorded as positive. Primary antibodies against Rab27B (1:200 dilution, Abcam, USA), E-cadherin, a-Catenin, Fibronectin, Vimentin (1:200 dilution, BD Transduction Laboratories, USA) were used in this study. The staining protocol used in this study was described previously [27].

\section{Measurements of Rab27B and Epithelial-mesenchymal transition (EMT) markers expression by IHC assay}

All sections were stained in DAB for the same amount of time. For each slide, five random fields were selected for scoring, and a mean score of each slide was used in final analyses. Positive staining was accessed by using a five-scale scoring system: 0 (no positive cells), $1 \quad(<25 \%$ positive cells), 2 ( $25 \%-50 \%$ positive cells), 3 (50\%-75\% positive cells), and 4 ( $>75 \%$ positive cells). To maintain objectivity, we used a four-scale scoring system to describe the intensity of positive staining: 0 (negative staining), 1 (weak staining, light yellow), 2 (moderate staining, yellow brown), and 3 (strong staining, brown). Rab27B expression index $=($ intensity score $)+($ positive score). Two independent pathologists without access to the clinicopathologic information performed the scorings. If all scorers assigned consistent results, then the value was selected. When completely different results were given, all of the scorers would work together to confirm a score.

\section{RNA extraction, reverse transcription and real-time PCR}

Total RNA was isolated from 12 pairs of $\mathrm{BC}$ tissues and their NAT tissue using TRIZOL reagent (Invitrogen). The extracted RNA was pretreated with RNAase-free 
DNase, and 2 ug RNA from each sample were used for cDNA synthesis primed with random hexamers. Realtime PCR was carried out using an ABI 7900HT fast real-time system (Applied Biosystems, Foster City, California, USA) to determine the expression pattern of Rab27B mRNA in each of the BC sample as well as the paired NAT tissue. Expression data were normalized to the geometric mean of the housekeeping gene glyceraldehydes 3-phosphate dehydrogenase (GAPDH). The first strand cDNA products were amplified with GAPDHspecific (F: 5'-CCACCCATGGCAAATTCCATGGCA-3' and R: 5'-TCTAGACGGCAGGTCAGGTCCACC-3') and Rab27B-specific (F: 5'- TGCGGGACAAGAGCGGTTC CG-3' and R: 5'- GCCAGTTCCCGAGCTTGCCGTT-3') primers by PCR.

\section{Western blot analysis}

Equal amounts of $\mathrm{BC}$ tissue lysates were resolved by SDS-polyacrylamide gel electrophoresis (SDS-PAGE) and electrotransferred to a polyvinylidene difluoride membrane (Pall Corp., Port Washington, NY). The tissues were then incubated with primary rabbit monoclonal antibodies against Rab27B (1:500 dilution, Abcam, USA). The immunoreactive signals were detected with an enhanced chemiluminescence kit (Amersham Biosciences, Uppsala, Sweden) used in accordance with the manufacturer's instructions.

\section{Selection of cutoff score}

Receiver operating characteristic (ROC) curve analysis was performed to determine the cutoff score for Rab27B high expression by using the 0,1-criterion [28]. At the Rab27B score, the sensitivity and specificity for each outcome under study was plotted, generating various ROC curves. The score selected as the cutoff value was that which was closest to the point with both maximum sensitivity and specificity. Tumors designated as having low expression of Rab27B were those with scores below or equal to the cutoff value, and tumors of high expression were those with scores above the value. To use ROC curve analysis, the clinicopathological characteristics were dichotomized as follows: tumor size stage (T1 vs. T2 + T3), pathologic grade (I vs. IIIII), lymph node metastasis (absent vs. present), clinical stage (I-II vs. III-IV), and survival status (death due to BC vs. censored).

\section{Statistical analysis}

The statistical significance of the correlation between Rab27B expression level and patient survival was estimated by using the Mantel-Cox log-rank test. ROC curve analysis was conducted to evaluate the predictive value of the parameters. The chi-square test or Fisher's exact test was used to evaluate the relationship between Rab27B expression and clinicopathological variables.
Spearman's rank correlation test was performed to evaluate the relationship between Rab27B and EMT markers expression by IHC. The multivariate Cox proportional hazards model was used to estimate the hazard ratios and 95\% confidence intervals of patient outcome. The relationships between the Rab27B expression levels and disease-specific survival (DSS) were determined by Kaplan-Meier analysis. Log-rank tests were performed to determine the difference in survival probabilities between patient subsets. All $P$-values quoted are twosided, and $P<0.05$ was considered to represent a statistically significant result. Statistical analysis was performed by using SPSS 16.0 software (Chicago, IL, USA).

\section{Consent}

Written informed consent was obtained from the patient for publication of this report and any accompanying images.

\section{Results}

\section{Expression pattern of Rab27B in BC tissues}

To investigate the status of Rab27B gene expression in $\mathrm{BC}$, we used Real-time PCR to measure the mRNA expression in 12 pairs of primary $\mathrm{BC}$ and NAT specimens. Compared with their NATs, 8 of 12 BC had upregulated expression. Consistently, western blot and IHC analysis showed that the 8 cases also had higher Rab27B protein expression than adjacent tissues. The BC cases with upregulated expression of Rab27B are shown in Figure $1 \mathrm{~A}$ and $1 \mathrm{~B}$. Interestingly, our IHC and Real-time PCR result in these 12 paired BC and NAT specimens demonstrated the protein and mRNA level of Rab27B are positively correlated $\left(r_{s}=0.705, P<0.001\right)$. IHC analysis showed that positive expression of Rab27B was primarily observed in the cell cytoplasm and membrane. Immunoreactivity of Rab27B protein in $\mathrm{BC}$ ranged from 0 to 7. In normal breast tissues and FA tissues, no or weak Rab27B staining was detected (Figure 1C-D). However, Rab27B showed more positive staining in DCIS tissues, BC tissues and metastatic LNs (Figure 1E-1G). An interesting phenomenon was that Rab27B expression appeared to increase incrementally with the magnitude of cancer progression in tissue $(P<0.05$. Figure $1 \mathrm{H})$.

\section{Selection of cutoff score for high expression of Rab27B}

The ROC curves for each clinicopathological feature define the cutoff point on the curve closet to the point $(0.0,1.0)$, which maximized both sensitivity and specificity for the outcome. Tumors with scores above the obtained cutoff value were considered as highlyexpressed Rab27B leading to the greatest number of tumors correctly classified as having or not having the clinical outcome. The corresponding area under the curve (AUC) and cutoff scores were collected and are 


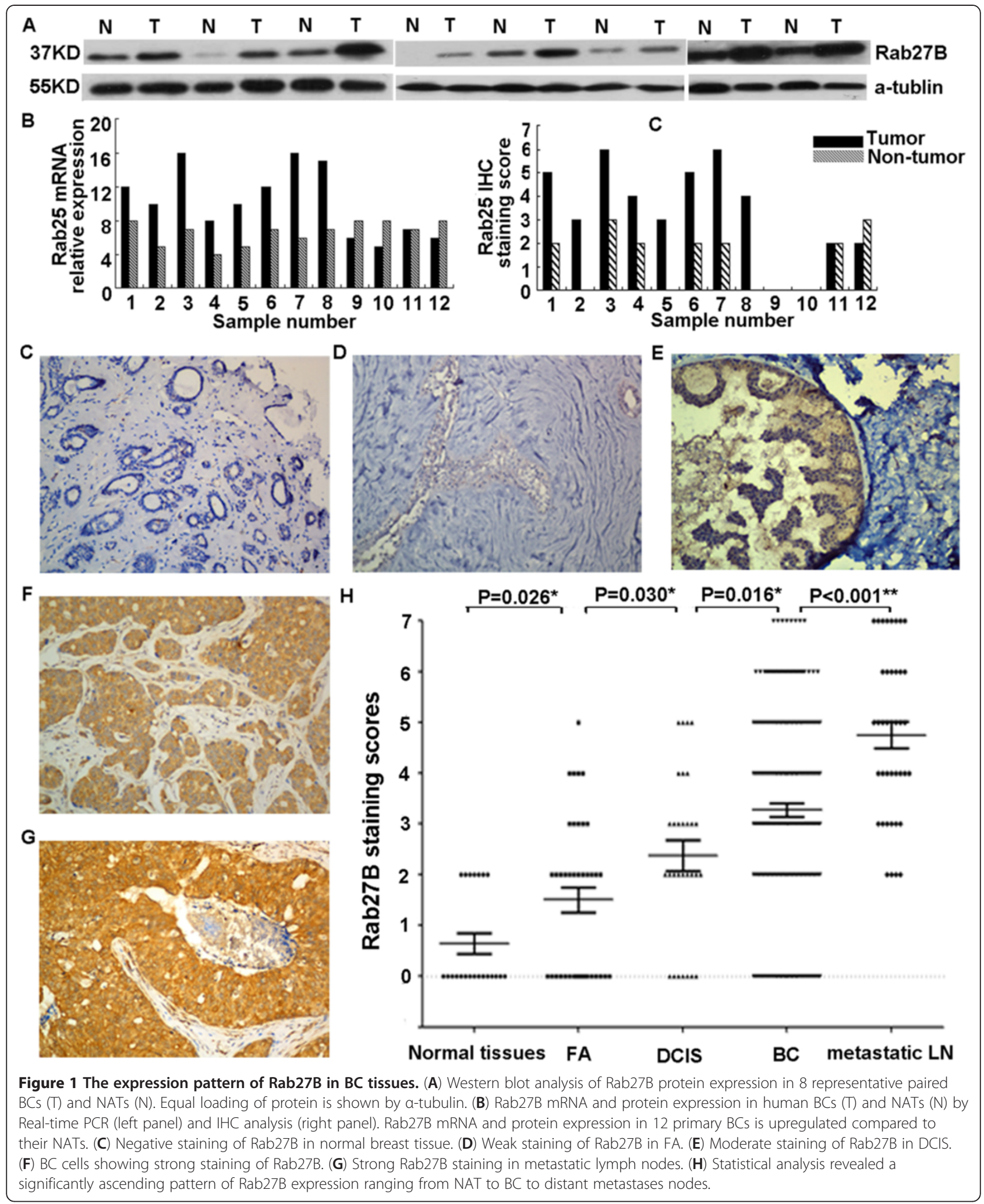


Table 1 The corresponding cutoff socre of Rab27B expression for each clinicapathological feature according to ROC curve analysis

\begin{tabular}{lccr}
\hline Variable & Subvariable & Cutoff score & $P$ value \\
\hline Histology grade & $I(n=47)$ vs. IIIII $(n=174)$ & $>3$ & $0.014^{*}$ \\
Tumor size stage & $T 1(n=63)$ vs. T2 $+T 3(n=158)$ & $>2$ & 0.917 \\
Lymph nodes metastasis & absent $(n=105)$ vs. present $(n=116)$ & $>2$ & 0.064 \\
Clinical stage & $|I|(n=181)$ vs. III $(n=40)$ & $>4$ & $0.046^{*}$ \\
Live status & Live $(n-171)$ vs. death $(n=50)$ & $>3$ & $<0.001^{*}$ \\
\hline
\end{tabular}

*Statistically significant difference.

shown in Additional file 1: Figure S1 and Table 1, respectively. In our current study, ROC curve analysis for living status had the shortest distance from the curve to the point (i.e., 0.0, 1.0), and we selected the cutoff value determined by living status. The sensitivity and specificity of Rab27B as a prognosis prediction marker of breast cancer patients are 88.2 and 63.5 respectively. Thus, the cutoff score for high expression of Rab27B was defined when a score greater than 3 was obtained in IHC analysis for Rab27B.

\section{Association of Rab27B expression with BC clinicopathologic features}

According to the cutoff value, high expression of Rab27B was observed in 0 of $22(0 \%)$ normal breast tissues, 5 of 40 (12.5\%) FA tissues, 10 of 30 (33.3\%) DCIS tissues, 107 of $221(48.4 \%)$ BCs, and 30 of $40(75.0 \%)$ metastatic LNs, respectively $(P<0.001$, "Chi-square test, Table 2$)$.

The rates of high expression of Rab27B in BCs with respect to several standard clinicopathological features are presented in Table 2. High expression of Rab27B was positively correlated with pathology grade, advanced clinical stage, lymph node metastasis, and ER status $(P<0.05$, Table 1$)$. There was no significant association between Rab27B expression and other clinicopathological features, such as patient age, menopausal status, T stage, RP status, or CerbB2 status $(P>0.05$, Table 3$)$.

Elevated Rab27B expression predicts poor prognosis of $B C$ Kaplan-Meier curves showed that, in the primary BC category, the cumulative 5-year disease-specific survival (DSS) rate was $95.6 \%$ for patients with lower levels of
Rab27B and $64.0 \%$ for patients with higher levels of Rab27B expression $(P<0.01$, Figure $2 \mathrm{~A})$. Furthermore, the expression levels of Rab27B were strongly correlated with patients' survival even after stratifying the patients based upon their clinicopathological variables. Subset analysis showed that high Rab27B expression had a decreased survival time regardless of clinical stage, tumor size stage, and lymph node metastasis $(P<0.05$, Figure $2 \mathrm{~B}-2 \mathrm{G})$.

\section{Multivariate survival analysis}

Cox regression proportional hazard analyses indicated that high expression of Rab27B was a significant risk factor for adverse DSS (hazard ratio, 8.661; 95\% confidence interval $[\mathrm{CI}], 3.897-19.251, P<0.001)$. Of the other variables, histology grade, clinical stage, tumor size stage, lymph node metastasis, and PR status were also found to be prognostic predictors of DSS (Table 4). Since clinical stage is a combination of tumor size stage and lymph node involvement, we only include tumor size stage and lymph node metastasis in the multivariate analysis. After multivariate analysis, the expression level of Rab27B was found to be a significant independent prognostic factor of poor DSS in BC patients (hazard ratio, 9.120; 95\% CI, 4.056-20.506, $P<0.001$ ). Histology grade, tumor size stage, lymph node metastasis, and negative PR status also independently predicted poor overall survival (Table 4).

\section{Correlation between the expression of Rab27B and EMT markers in $\mathrm{BC}$ tissues}

To explore the relationship of Rab27B and EMT process, IHC staining of was performed in 221 primary

Table 2 The expression of Rab27B in normal breast tissues and in benign and malignant breast tumors

\begin{tabular}{lccc}
\hline & Cases & \multicolumn{2}{c}{ Rab27B expression } \\
\cline { 3 - 4 } & & Low expression & High expression \\
\hline Normal breast tissues & 22 & $22(100 \%)$ & $0(0 \%)$ \\
Fibroadenoma of breast & 40 & $35(87.5 \%)$ & $5(12.5 \%)$ \\
Ductal carcinoma in situ & 30 & $23(76.7 \%)$ & $7(23.3 \%)$ \\
Invasive breast cancer & 221 & $114(51.6 \%)$ & $107(48.4 \%)$ \\
Metastatic lymph nodes & 40 & $10(25.0 \%)$ & $30(75.0 \%)$ \\
\hline
\end{tabular}

*Statistically significant difference. 
Table 3 Relationship between Rab27B expression level and clinicopathologic parameters of BC

\begin{tabular}{|c|c|c|c|c|}
\hline \multirow[t]{2}{*}{ Variable } & \multirow[t]{2}{*}{ Number of cases } & \multicolumn{2}{|c|}{ Rab27B expression } & \multirow[t]{2}{*}{$P$ value } \\
\hline & & High expression & Low expression & \\
\hline \multicolumn{5}{|l|}{ Age (years) } \\
\hline$\geq 47^{\mathrm{a}}$ & 103 & $46(44.7 \%)$ & $57(55.3 \%)$ & \\
\hline$<47$ & 118 & $61(51.7 \%)$ & $57(48.3 \%)$ & 0.297 \\
\hline \multicolumn{5}{|c|}{ Pathologic grade } \\
\hline । & 47 & 15(31.9\%) & $32(69.1 \%)$ & \\
\hline\|\|$\|$ & 174 & $92(52.9 \%)$ & $82(47.1 \%)$ & $0.011^{*}$ \\
\hline \multicolumn{5}{|l|}{ Clinical stage } \\
\hline III & 181 & $80(44.2 \%)$ & $101(55.8 \%)$ & \\
\hline III & 40 & $27(67.5 \%)$ & $13(32.5 \%)$ & $0.008^{*}$ \\
\hline \multicolumn{5}{|c|}{ Tumor size stage } \\
\hline $\mathrm{T} 1$ & 63 & $32(50.8 \%)$ & $31(49.2 \%)$ & \\
\hline $\mathrm{T} 2+\mathrm{T} 3$ & 158 & $82(51.9 \%)$ & $76(48.1 \%)$ & 0.883 \\
\hline \multicolumn{5}{|c|}{ Menopausal status } \\
\hline Absent & 90 & $53(58.9 \%)$ & $37(41.1 \%)$ & \\
\hline Present & 131 & $61(46.6 \%)$ & $70(53.4 \%)$ & 0.072 \\
\hline \multicolumn{5}{|c|}{ Lymph node metastasis } \\
\hline Metastasis & 116 & $65(56.0 \%)$ & $51(44.0 \%)$ & \\
\hline No metastasis & 105 & $42(40.0 \%)$ & $63(60.0 \%)$ & $0.021^{*}$ \\
\hline \multicolumn{5}{|l|}{ ER status } \\
\hline Negative & 97 & $37(38.1 \%)$ & $60(61.9 \%)$ & \\
\hline Positive & 124 & $70(56.5 \%)$ & $54(43.5 \%)$ & $0.007^{*}$ \\
\hline \multicolumn{5}{|l|}{ PR status } \\
\hline Negative & 91 & $39(42.9 \%)$ & $52(57.1 \%)$ & \\
\hline Positive & 130 & $68(52.3 \%)$ & $62(47.7 \%)$ & 0.166 \\
\hline \multicolumn{5}{|l|}{ CerbB2 status } \\
\hline $0,1+, 2+$ & 147 & $74(50.3 \%)$ & 73(49.7\%) & \\
\hline $3+$ & 74 & $33(44.6 \%)$ & $41(55.4 \%)$ & 0.42 \\
\hline \multicolumn{5}{|l|}{ Living status } \\
\hline Live & 170 & 63(37.1\%) & 107(62.9\%) & \\
\hline Dead & 51 & $44(86.3 \%)$ & 7(13.7\%) & $<0.001^{*}$ \\
\hline
\end{tabular}

BC breast cancer. a Mean age. *Statistically significant difference.

BC tissues. Representative IHC staining of EMT markers in $\mathrm{BC}$ tissues is shown in Figure 3. We observed that Rab27B expression level was positively correlated with the expression level of mesenchymal markers: Vimentin $\left(\mathrm{r}_{\mathrm{s}}=0.289, P<0.001, \mathrm{n}=221\right.$, Spearman's correlation analysis), Fibronectin $\left(\mathrm{r}_{\mathrm{s}}=0.327, P<0.001\right.$, $\mathrm{n}=221$, Spearman's correlation analysis), and inversely correlated with epithelial markers: E-cadherin $\left(\mathrm{r}_{\mathrm{s}}=-0.226\right.$, $P=0.001, \mathrm{n}=221$, Spearman's correlation analysis), $\beta$-catenin $\left(\mathrm{r}_{\mathrm{s}}=-0.389, \quad P<0.001, \mathrm{n}=221\right.$, Spearman's correlation analysis).These data illustrated that overexpression of Rab27B contributed to the EMT process in $\mathrm{BC}$ progression.

\section{Discussion}

Rab27B, a regulator of vesicle exocytosis, has been suggested to function as an oncogene in ER-positive breast cell lines both in vitro and in vivo [23]. However, without patients' survival information, we could not investigate the effect of Rab27B expression on patient prognosis. We performed the present study to investigate the expression dynamics of Rab27B and their clinicopathologic/prognostic significance in $\mathrm{BC}$ patients. To the best of our knowledge, this is the first study investigating the expression of Rab27B in a large series of BC patients.

The previous study showed that Rab27B is upregulated in BC [23]; however, Dong et.al reported that the 


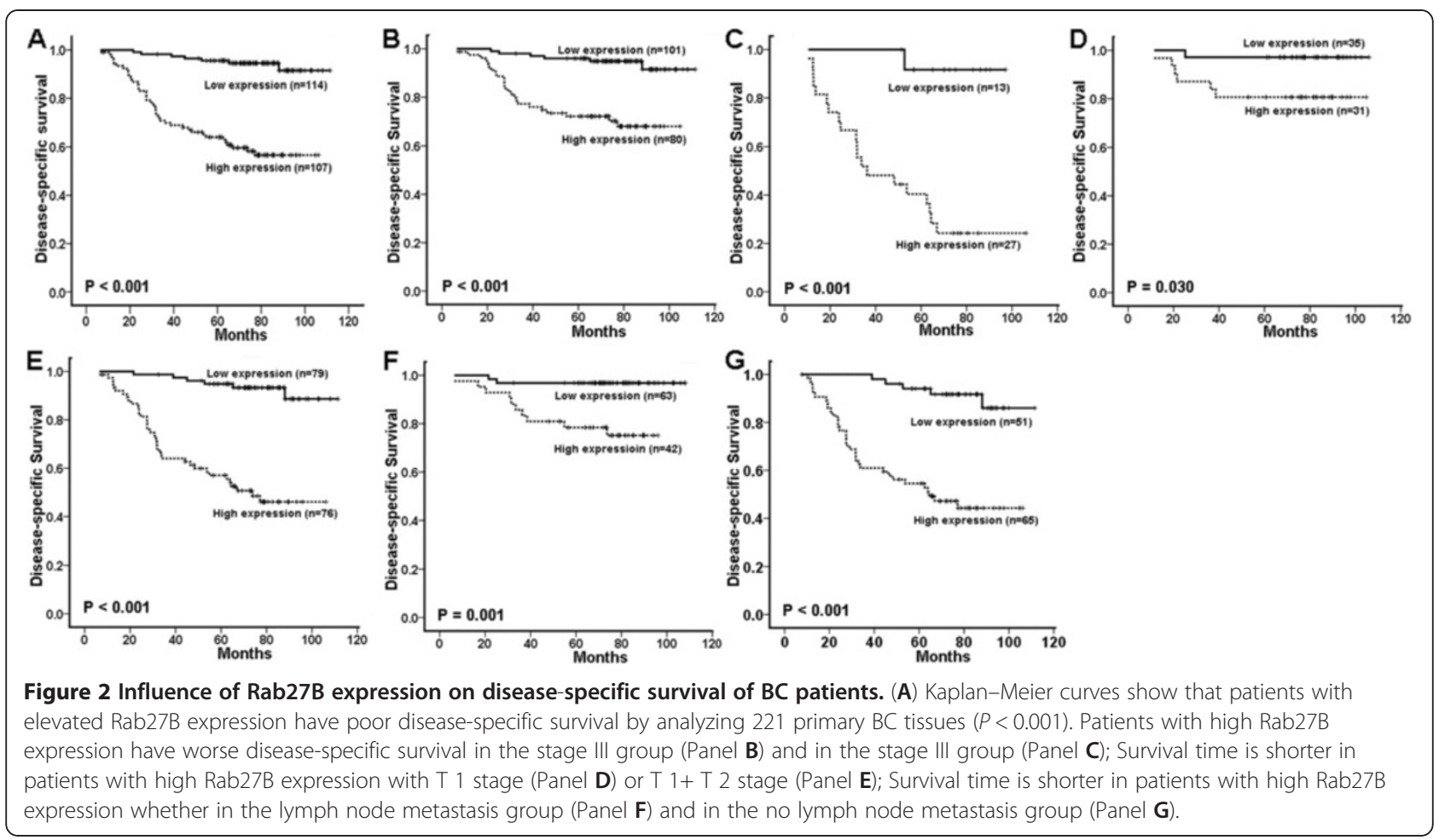

Rab27B expression level was lower in primary hepatocellular carcinoma than in matched adjacent tissues [29]. Our results showed that both the mRNA and protein levels were upregulated in BCs compared with their NATs. Furthermore, we are first to report that expression levels of Rab27B appeared to increase with the magnitude of cancer progression: a significant increasing expression of Rab27B was observed from normal breast tissue, to FA, DCIS, and to invasive $\mathrm{BC}$, metastatic LNs In our study, further correlation analysis revealed for the first time that high Rab27B expression was closely correlated with an aggressive phenotype of $\mathrm{BC}$, including ascending pathologic grade, advanced clinical stage, and lymph node metastasis. These data, in agreement with data of the previous study [23], indicate that increased Rab27B expression corresponds to the progressive magnitude of $\mathrm{BC}$ and might facilitate the invasive/metastatic phenotypes of this malignancy, Rab27B is responsible for regulating many secretory mechanisms. Many studies have demonstrated that tumor cells use exosomes to communicate with surrounding tissues and immune cells, creating an immunosuppressive microenvironment for tumor progression [30-33]. Thus, we propose that Rab27B is associated with tumor progression because of its function as a transport vesicle.

It is well known that tumor invasion and metastases are responsible for most cancer-related mortality. As for invasive $\mathrm{BC}$, the lymphatic system is the primary pathway to metastatic disease. For patients with lymph node metastasis, additional chemoradiation is required. Thus, identification of biomarkers that can be used to define the metastatic potential of $\mathrm{BC}$ may facilitate the development of appropriate therapeautic strategy earlier in the course of this cancer. In this study, we found that those with higher Rab27B expression are prone to have lymph node metastasis. We recommend that they be identified as patients at high risk in the clinic and that more radical therapy regimens should be delivered to them. Furthermore, the accuracy of predicting lymph node metastasis by examining Rab27B expression level (high vs. low) in our cohort is $60.7 \%$ (65/107). In light of these findings, we hypothesize that Rab27B may be a novel predictor of lymph node metastasis in $\mathrm{BC}$ patients. To address this issue, a further study in a larger cohort of $\mathrm{BC}$ patients is now underway.

As for the underlying mechanism involved in Rab27Bregulating $\mathrm{BC}$ invasive and metastasis potential, here we focused on the EMT process. During this process, epithelia cells lose their epithelia adherence, cell-cell contact and their polarity, and undergo remarkable remodeling of the cytoskeleton, all of which facilitate cell invasion and migration [34,35]. Interestingly, our results demonstrated that BCs with Rab27B high expression displayed the enhanced expression of the mesenchymal markers vimentin and fibronectin, and decreased expression of the epithelial markers E-cadherin and $\beta$-catenin, suggesting an EMT process during Rab27B-regulating $\mathrm{BC}$ development. The acquisition of EMT characteristics 
Table 4 Results of univariate and multivariate Cox proportional-hazards analysis for disease-specific survival

\begin{tabular}{|c|c|c|c|c|}
\hline \multirow[t]{2}{*}{ Variable } & \multirow[t]{2}{*}{ Subvariable } & \multicolumn{3}{|c|}{ All cases $(n=221)$} \\
\hline & & $\mathrm{HR}$ & $95 \% \mathrm{Cl}$ & $P$-value \\
\hline \multicolumn{5}{|l|}{ Univariate } \\
\hline Age & $\geq 47^{\mathrm{a}}$ yr $(n=103)$ vs. $<47 \mathrm{yr}(n=117)$ & 1.654 & $0.931-2.938$ & 0.086 \\
\hline Histology grade & I $(n=47)$ vs. |||| $\mid(n=174)$ & 7.696 & $1.871-31.651$ & $0.005^{*}$ \\
\hline Clinical stage & I || (n=181) vs. III $(n=40)$ & 4.059 & $2.318-7.016$ & $<0.001^{*}$ \\
\hline Tumor size stage & $T 1(n=63)$ vs. $T 2+T 3(n=158)$ & 2.799 & $1.260-6.219$ & $0.011^{*}$ \\
\hline Lymph nodes metastasis & absent $(n=105)$ vs. present $(n=116)$ & 3.361 & $1.759-6.422$ & $<0.001^{*}$ \\
\hline Menopausal status & absent $(n=131)$ vs. present $(n=90)$ & 1.549 & $0.857-2.798$ & 0.147 \\
\hline Rab27B expression & low $(n=107)$ vs. high $(n=114)$ & 8.661 & $3.897-19.251$ & $<0.001^{*}$ \\
\hline ER status & absent $(==97)$ vs. present $(n=124)$ & 0.588 & $0.339-1.022$ & 0.06 \\
\hline PR status & absent $(n=91)$ vs. present $(n=130)$ & 0.505 & $0.291-0.878$ & $0.015^{*}$ \\
\hline CerbB2 status & $0,1+, 2+(n=147)$ vs. $3+(n=74)$ & 1.504 & $0.861-2.628$ & 0.152 \\
\hline \multicolumn{5}{|l|}{ Multivariate } \\
\hline Histology grade & I $(n=47)$ vs. |||| $\mid(n=174)$ & 4.808 & $1.154-20.030$ & $0.031^{*}$ \\
\hline Tumour size & $T 1(n=63)$ vs. $T 2+T 3(n=158)$ & 2.630 & $1.167-5.924$ & $0.020^{*}$ \\
\hline Lynph nodes metastasis & absent $(n=105)$ vs. present $(n=116)$ & 2.696 & $1.398-5.197$ & $0.003^{*}$ \\
\hline Rab27B expression & low $(n=107)$ vs. high $(n=114)$ & 9.120 & $4.056-20.506$ & $<0.001^{*}$ \\
\hline PR status & absent $(n=91)$ vs. present $(n=130)$ & 0.412 & $0.233-0.729$ & $0.002^{*}$ \\
\hline
\end{tabular}

${ }^{a}$ Mean age. Cox propotional hazard regression model, enter; HR, Hazard ratio; $\mathrm{Cl}$, confidence interval; *Statistically significant difference.

may give these $\mathrm{BC}$ tumor cells a higher aggressive potential, resulting in the invasive and metastatic behavior. Thus we underscore that the EMT process might be a potential underlying mechanism in Rab27B-regulating $\mathrm{BC}$ invasion and metastasis. However, further study is underway to identify the special pathway involved in Rab27B mediated EMT process in BC.

Recently Rab27B was identified as a predictor of prognosis in HCC [29]. With regard to the prognostic effect of Rab27B in BC, our findings show for the first time that $\mathrm{BC}$ patients with elevated Rab27B expression had worse survival outcomes than those expressing lower
Rab27B, suggesting the clinical value of Rab27B in assessing the prognosis of $\mathrm{BC}$ patients. Furthermore, even after by stratified analysis, Rab27B could display a favorable prognosis value in each subgroup. Our findings strongly suggest that Rab27B may be a novel and important prognostic marker for $\mathrm{BC}$ patients.

It is noteworthy that the previous study found no Rab27B staining in ER-negative samples [23]; however, we detected positive staining in such samples. The difference may reflect differences in Rab27B status in the samples used in different studies, which obtained tissue samples from different populations. Furthermore, the

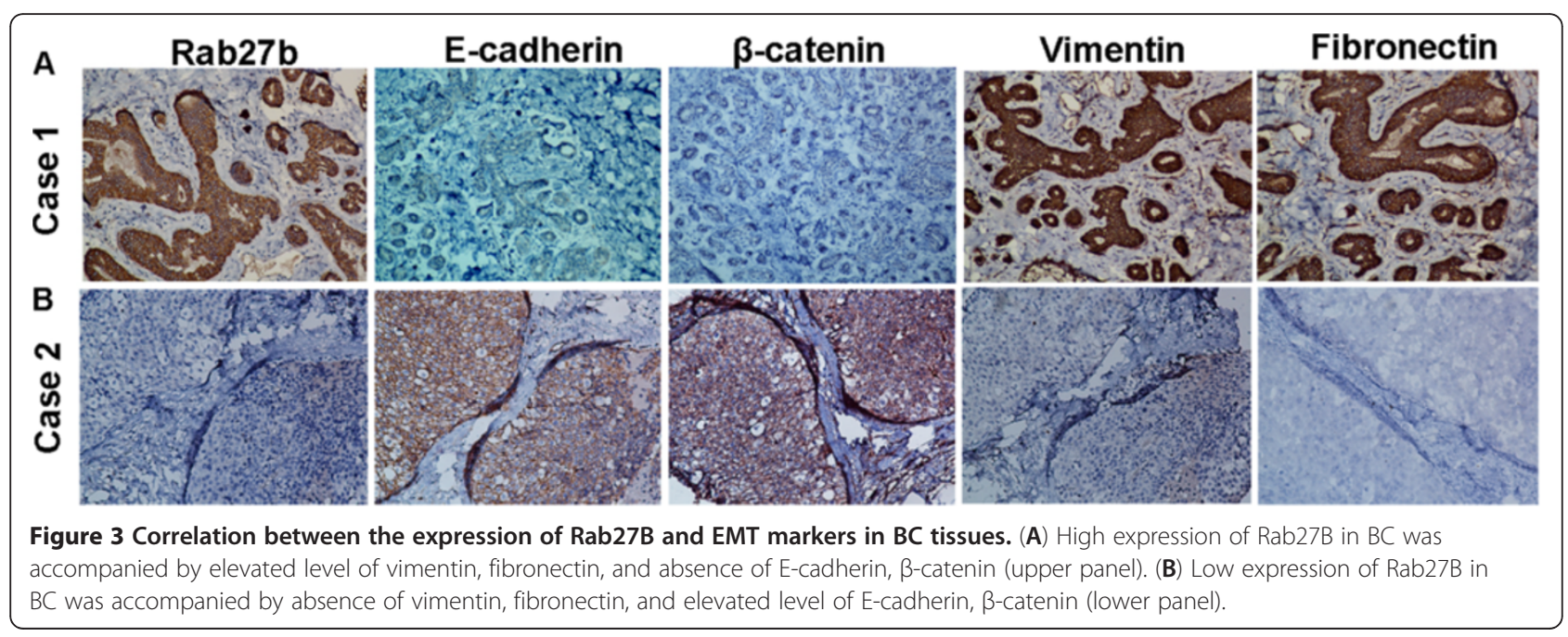


previous study enrolled only 59 cases, whereas 221 are included in our study, leading to a more convincing result [23]. Furthermore, in our enrolled cases, the prognostic significance of Rab27B expression was not confined to this group. These results confirm that the pro-oncogenic function of Rab27B is not ER-dependent. Thus, Rab27B could be adopted as a widely used biomarker in predicting patients' survival. However, these results should be confirmed in a large, multicenter trial.

\section{Conclusion}

In summary, our data suggest that increased expression of Rab27B, assocatied with elevated mesenchymal EMT markers, is related to the aggressive and metastatic potential of $\mathrm{BC}$ and thus contributes to a poor prognostic phenotype. Examining Rab27B expression by IHC analysis, is an effective way to assess $\mathrm{BC}$ patients' survival outcome. This tool could be of great value to help clinicians to make an optimal individual treatment for $\mathrm{BC}$ patients.

\section{Additional file}

Additional file 1: Figure S1. Receiver operating characteristic curve analysis was used to determine the cutoff score for the high expression of Rab27B. The sensitivity and specificity scores of each outcome were plotted: (A) Histology grade $(P=0.014)$ (B) Tumor size stage $(P=0.917)$; (C) Lymph node metastasis status $(P=0.064)$; (D) Clinical stage $(P=0.046)$; (E) Survival status $(P<0.001)$.

\section{Abbreviations}

BC: Breast cancer; FA: Fibroadenoma of breast; DCIS: Ductal carcinoma in situ; LN: Lymph node; NAT: Adjacent noncancerous tissues; IHC: Immunohistochemistry staining; ER: Eestrogen receptor; PR: Progesterone receptor; HER-2: Human epidermal growth factor receptor 2; DSS: Disease-specific survival; ROC: The receiver operating characteristic curve; EMT: Epithelial-mesenchymal transition.

\section{Competing interests}

The authors declare that they have no conflict of interest.

\section{Authors' contributions}

SJM, DX and YXZ carried out and coordinated the study. JXZ, XXH, ZTT, DQ YJL, HXD, MBC, DZL and MYH performed the experiments. JXZ and XXH analyzed the data. JXZ wrote the paper. All authors read and approved the final manuscript.

\section{Acknowledgements}

This work was supported by grants from the 973 Project of China (2010CB912802 and 2010CB529404), the National Natural Science Foundation of China (No. 81072222 and 81172340), the Natural Science Foundation of Guangdong (No. S2011020002762) and the Ph.D. Programs Foundation of Ministry of Education of China (20110171110078).

\section{Author details}

'State Key Laboratory of Oncology in South China, Cancer Center, Sun YatSen University, 651\# Dongfeng Road East, Guangzhou 510060, China. ${ }^{2}$ Department of Pathology, Cancer Center, Sun Yat-Sen University, 651\# Dongfeng Road East, Guangzhou 510060, China. ${ }^{3}$ Cancer Center, The First Affiliated Hospital, University of South China, Hengyang, China. ${ }^{4}$ Department of Radiotherapy, The First Affiliated Hospital, Anhui Medical University, Hefei, China.
Received: 24 August 2012 Accepted: 28 November 2012

Published: 5 December 2012

\section{References}

1. Yang $L, L i L D$, Chen YD, Parkin DM: Time trends, estimates and projects for breast cancer incidence and mortality in China. Zhonghua Zhong Liu Za Zhi 2006, 28:438-440.

2. Parkin DM, Bray F, Ferlay J, Pisani P: Global cancer statistics, 2002. CA Cancer J Clin 2005, 55:74-108.

3. Bombonati A, Sgroi DC: The molecular pathology of breast cancer progression. J Pathol 2011, 223:307-317.

4. Jemal A, Siegel R, Ward E, Hao Y, Xu J, Murray T, Thun MJ: Cancer statistics, 2008. CA Cancer J Clin 2008, 58:71-96.

5. Harris L, Fritsche H, Mennel R, Norton L, Ravdin P, Taube S, Somerfield MR, Hayes DF, Bast RC: American society of clinical oncology 2007 update of recommendations for the use of tumor markers in breast cancer. J Clin Oncol 2007, 25:5287-5312.

6. Fukuda M: Regulation of secretory vesicle traffic by Rab small GTPases. Cell Mol Life Sci 2008, 65:2801-2813.

7. Pereira-Leal JB, Seabra MC: Evolution of the Rab family of small GTP-binding proteins. J Mol Biol 2001, 313:889-901.

8. Zerial M, McBride H: Rab proteins as membrane organizers. Nat Rev Mol Cell Biol 2001, 2:107-117.

9. Pfeffer SR: Structural clues to Rab GTPase functional diversity. J Biol Chem 2005, 280:15485-15488.

10. Stenmark H: Rab GTPases as coordinators of vesicle traffic. Nat Rev Mol Cell Biol 2009, 10:513-525.

11. Chen D, Guo J, Miki T, Tachibana M, Gahl WA: Molecular cloning and characterization of rab27a and rab27b, novel human rab proteins shared by melanocytes and platelets. Biochem Mol Med 1997, 60:27-37.

12. Westbroek W, Lambert J, De Schepper S, Kleta R, Van Den Bossche K, Seabra MC, Huizing M, Mommaas M, Naeyaert JM: Rab27b is up-regulated in human Griscelli syndrome type II melanocytes and linked to the actin cytoskeleton via exon F-Myosin Va transcripts. Pigment Cell Res 2004, 17:498-505.

13. Jordens I, Westbroek W, Marsman M, Rocha N, Mommaas M, Huizing M, Lambert J, Naeyaert JM, Neefjes J: Rab7 and Rab27a control two motor protein activities involved in melanosomal transport. Pigment Cell Res 2006, 19:412-423.

14. Westbroek W, Tuchman M, Tinloy B, De Wever O, Vilboux T, Hertz JM, Hasle H, Heilmann C, Helip-Wooley A, Kleta R, Gahl WA: A novel missense mutation (G43S) in the switch I region of Rab27A causing griscelli syndrome. Mol Genet Metab 2008, 94:248-254.

15. Cheng KW, Lahad JP, Kuo WL, Lapuk A, Yamada K, Auersperg N, Liu J, Smith-McCune K, Lu KH, Fishman D, Gray JW, Mills GB: The RAB25 small GTPase determines aggressiveness of ovarian and breast cancers. Nat Med 2004, 10:1251-1256.

16. Kanda I, Nishimura N, Nakatsuji H, Yamamura R, Nakanishi H, Sasaki T: Involvement of Rab13 and JRAB/MICAL-L2 in epithelial cell scattering. Oncogene 2008, 27:1687-1695.

17. Liu YJ, Wang Q, Li W, Huang XH, Zhen MC, Huang SH, Chen LZ, Xue L, Zhang HW: Rab23 is a potential biological target for treating hepatocellular carcinoma. World J Gastroenterol 2007, 13:1010-1017.

18. Hou Q, Wu YH, Grabsch H, Zhu Y, Leong SH, Ganesan K, Cross D, Tan LK Tao J, Gopalakrishnan V, Tang BL, Kon OL, Tan P: Integrative genomics identifies RAB23 as an invasion mediator gene in diffuse-type gastric cancer. Cancer Res 2008, 68:4623-4630.

19. Fukui K, Tamura S, Wada A, Kamada Y, Igura T, Kiso S, Hayashi N: Expression of Rab5a in hepatocellular carcinoma: possible involvement in epidermal growth factor signaling. Hepatol Res 2007, 37:957-965

20. Bravo-Cordero JJ, Marrero-Diaz R, Megias D, Genis L, Garcia-Grande A, Garcia MA, Arroyo AG, Montoya MC: MT1-MMP proinvasive activity is regulated by a novel Rab8-dependent exocytic pathway. EMBO J 2007, 26:1499-1510.

21. Wang JS, Wang FB, Zhang QG, Shen ZZ, Shao ZM: Enhanced expression of Rab27A gene by breast cancer cells promoting invasiveness and the metastasis potential by secretion of insulin-like growth factor-II. Mol Cancer Res 2008, 6:372-382.

22. Chia WJ, Tang BL: Emerging roles for Rab family GTPases in human cancer. Biochim Biophys Acta 2009, 1795:110-116. 
23. Hendrix A, Maynard D, Pauwels P, Breams $G$, Denys $H$, Van den Broecke $R$, Lambert J, Van Belle S, Cocquyt V, Gespach C, Brache M, Seabra MC, Gahl WA, De Wever O, Westbroek W: Effect of the secretory small GTPase Rab27B on breast cancer growth, invasion, and metastasis. J Natl Cancer Inst 2010, 102:866-880.

24. Singletary SE, Allred C, Ashley P, Bassett LW, Berry D, Bland KI, Borgen PI, Clark GM, Edge SB, Hayes DF, Hughes LL, Hutter RV, Morrow M, Page DL, Recht A, Theriault RL, Thor A, Weaver DL, Wieand HS, Greene FL: Staging system for breast cancer: revisions for the 6th edition of the AJCC cancer staging manual. Surg Clin North Am 2003, 83:803-819.

25. Yan LX, Wu QN, Zhang Y, Li YY, Liao DZ, Hou JH, Fu J, Zeng MS, Yun JP, Wu QL, Zeng YX, Shao JY: Knockdown of miR-21 in human breast cancer cell lines inhibits proliferation, in vitro migration and in vivo tumor growth. Breast Cancer Res 2011, 13:R2.

26. Zhang Y, Yan LX, Wu QN, Du ZM, Chen J, Liao DZ, Huang MY, Hou JH, Wu QL, Zeng MS, Huang WL, Zeng YX, Shao JY: miR-125b is methylated and functions as a tumor suppressor by regulating the ETS1 proto-oncogene in human invasive breast cancer. Cancer Res 2011, 71:3552-3562

27. Yan LX, Huang XF, Shao Q, Huang MY, Deng L, Wu QL, Zeng YX, Shao JY: MicroRNA miR-21 overexpression in human breast cancer is associated with advanced clinical stage, lymph node metastasis and patient poor prognosis. RNA 2008, 14:2348-2360.

28. Zlobec I, Steele R, Terracciano L, Jass JR, Lugli A: Selecting immunohistochemical cut-off scores for novel biomarkers of progression and survival in colorectal cancer. J Clin Pathol 2007, 60:1112-1116.

29. Dong WW, Mou Q, Chen J, Cui JT, Li WM, Xiao WH: Differential expression of Rab27A/B correlates with clinical outcome in hepatocellular carcinoma. World J Gastroenterol 2012, 18:1806-1813

30. Mignot G, Chalmin F, Ladoire S, Rebe C, Ghiringhelli F: Tumor exosome-mediated MDSC activation. Am J Pathol 2011, 178:1403-1404. author reply 1404-1405.

31. Ochieng J, Pratap S, Khatua AK, Sakwe AM: Anchorage-independent growth of breast carcinoma cells is mediated by serum exosomes. Exp Cell Res 2009, 315:1875-1888.

32. Hegmans JP, Bard MP, Hemmes A, Luider TM, Kleijmeer MJ, Prins JB, Zitvogel L, Burgers SA, Hoogsteden HC, Lambrecht BN: Proteomic analysis of exosomes secreted by human mesothelioma cells. Am J Pathol 2004, 164:1807-1815.

33. Cho JA, Park H, Lim EH, Lee KW: Exosomes from breast cancer cells can convert adipose tissue-derived mesenchymal stem cells into myofibroblast-like cells. Int J Oncol 2012, 40:130-138.

34. Arias AM: Epithelial mesenchymal interactions in cancer and development. Cell 2001, 105:425-431.

35. Thiery JP: Epithelial-mesenchymal transitions in tumour progression. Nat Rev Cancer 2002, 2:442-454

doi:10.1186/1479-5876-10-242

Cite this article as: Zhang et al:: Overexpression of the secretory small GTPase Rab27B in human breast cancer correlates closely with lymph node metastasis and predicts poor prognosis. Journal of Translational Medicine 2012 10:242.

\section{Submit your next manuscript to BioMed Central and take full advantage of:}

- Convenient online submission

- Thorough peer review

- No space constraints or color figure charges

- Immediate publication on acceptance

- Inclusion in PubMed, CAS, Scopus and Google Scholar

- Research which is freely available for redistribution 